\title{
Cell-Substrate Associations during the Amoeboid Locomotion of Naegleria
}

\author{
By TERRY M. PRESTON AND CONRAD A. KING \\ Zoology Department, University College London, Gower Street, London WC1E 6BT
}

(Received 16 August 1977)

\section{INTRODUCTION}

Experimental investigations into the phenomenon of amoeboid locomotion have largely focused on the problem of force generation in cytoplasmic streaming and the production of pseudopodia (Wohlfarth-Botterman, 1973). Meanwhile, the behaviour of an amoeba's surface with respect to the substrate has received relatively little consideration (Dellinger, 1906; Bell \& Jeon, 1963; Haberey, 1971). This is surprising since the dynamic behaviour of the ventral side of an amoeba is crucial to locomotion, for the cell has to be able to make and subsequently break adhesions to a plane surface in order to progress across it. The precise mechanism of this temporary association with non-living substrates has long remained a matter of conjecture principally because of lack of evidence relating to the nature of the physical forces involved. Nevertheless one might hope to seek an explanation among those hypotheses already proposed to account for cell-cell adhesions, e.g. bridging systems, electrostatic interactions or interactions involving long-range forces (reviewed by Curtis, 1973).

The organism chosen for our experiments was Naegleria gruberi, a soil-dwelling amoeboflagellate that flourishes under conditions of very low ionic strength and displays a predominantly monopodial 'limax' pattern of locomotion. By means of reflexion interference microscopy (Curtis, 1964) we have been able to make direct observations on the general features of the undersurface of amoebae. This has resulted in two main findings which are the subject of this paper. Firstly, amoeboid locomotion requires a characteristic association with the substrate involving two types of contact which are distinct in their behaviour and degree of separation from that substrate. Secondly, we have discovered that the intervening gap between the major part of the underside of Naegleria and a surface across which it is moving can be profoundly influenced by the ionic conditions of the surrounding medium.

\section{METHODS}

Organism. Naegleria gruberi (stock 1518/1c, Culture Collection of Algae and Protozoa, Cambridge) was maintained at $25^{\circ} \mathrm{C}$ in monoxenic culture with Escherichia coli on $0.2 \%(\mathrm{w} / \mathrm{v})$ peptone agar plates. The protozoa were harvested in 2 mM-Tris $/ \mathrm{HCl} \mathrm{pH} \mathrm{7.4} \mathrm{and} \mathrm{washed} \mathrm{several} \mathrm{times} \mathrm{to} \mathrm{remove} \mathrm{most} \mathrm{of} \mathrm{the} \mathrm{bacteria.}$ An estimate of cell number was made with a Coulter counter (model $\mathbf{Z B}_{\mathrm{I}}$ ). The amoebae were then suspended in deionized water $\left(0.6 \mu \mathrm{S}\right.$ specific conductivity) to give $10^{7}$ cells $\mathrm{ml}^{-1}$. A $100 \mu \mathrm{l}$ sample of this suspension was introduced into a perfusion chamber, the windows of which were glass coverslips that had been cleaned with chromic acid. After the cells had settled on the lower coverslip the chamber was inverted and perfused with deionized water to remove unattached cells and any debris. The chamber was then locked into position on the stage of the interference reflexion microscope. Individual amoebae could be observed whilst different solutions were drawn through the chamber by a peristaltic pump.

Chemicals. $\mathrm{KCl}$ and sucrose (analytical reagent grade) were from $\mathrm{BDH}$.

Reflexion interference microscopy. Since this is not a common method of microscopy, a brief summary of the optical principles will be given. Amoebae in deionized water are allowed to settle on a coverglass which is then inverted and viewed by epi-illumination through an oil immersion objective lens; this also functions as a condenser. Some of the incident collimated light will be reflected $\left(R_{1}\right)$ at the coverglass-water boundary due 
to the change in refractive index there. A proportion of light waves transmitted through the water will be similarly reflected $\left(R_{2}\right)$ on striking the ventral surface of a cell. If this intervening gap is less than $1000 \mathrm{~nm}$, the optical path difference $(\Delta)$ between the waves $R_{1}$ and $R_{2}$ is such that in white light they will interact to produce interference colours. White light interference colours occur in a particular sequence directly related to $\Delta$, which can thus be estimated by reference to a standard colour chart. (The optical path here is analogous to that responsible for producing Newton's rings by reflexion.) At a $\Delta$ value of less than $40 \mathrm{~nm}$, a black image is obtained. As $\Delta$ increases, the image changes through grey to white. For values of $\Delta$ greater than $300 \mathrm{~nm}$ chromatic images of the standard interference colour series will be encountered.

A Vickers Photoplan stand was used with a Zeiss $\times 100$ planapochromat oil immersion lens. White light illumination was provided by a $100 \mathrm{~W}$ quartz halogen source. Green light was selected from the output of a $200 \mathrm{~W}$ mercury lamp by a Balzers K4 interference filter. The immersion lens was used at an incident numerical aperture (I.N.A.) of 1.0, calculated according to the method of Izzard \& Lochner (1976). A television camera (Link 106; Andover, Hampshire), connected to a videotape recorder, was positioned on the microscope to monitor the locomotory behaviour of amoebae during experiments. Figs $1(a, b, c)$ were produced by taking $35 \mathrm{~mm}$ photographs of the recordings on the television monitor.

Scanning electron microscopy. Suspensions of amoebae were allowed to settle on the surface of chromic acid-cleaned coverslips for $5 \mathrm{~min}$ at room temperature before fixation in $1 \%(\mathrm{w} / \mathrm{v}) \mathrm{OsO}_{4}$. The attached cells were dehydrated by means of a graded series of acetone-water mixtures before critical point drying. The specimens were coated with carbon and then gold before being examined in a Cambridge S4-10 microscope at $5 \mathrm{kV}$. Ilford FP4 $35 \mathrm{~mm}$ film was used for photographic records.

\section{RESULTS AND DISCUSSION}

The white light interference pattern produced in deionized water by the undersurface of Naegleria amoebae was achromatic (i.e. zero order) consisting of two distinct parts. The major component of the interference image, which could involve a large amount of the midventral face of the cell, was white to whitish-grey and extremely protean in form (Fig. $1 a$ ). We term this area 'associated contact'. Contrasting with the instability of the 'associated contacts' were points of closest approach which, by analogy with fibroblasts, we shall call 'focal contacts'. These were evident as black spots roughly $0.4 \mu \mathrm{m}$ in diameter that always formed from areas of 'associated contact'. The lack of interference contours about the 'focal contacts' suggested that these were the tips of cylindrical or narrowly tapering filopodia subtended from the ventral surface of the amoeba. Therefore the 'associated contacts' forming at a distance from the substrate functioned as a platform from which fine cytoplasmic extensions could be dispatched to form the 'focal contacts'. Once established, the 'focal contacts' were stable adhesions. Because of this, translational movement of an amoeba resulted in elongation of these filopodia and their extension from the cell's posterior region (uroid). On reaching several micrometres in length these trailing filopodia, visible now by phase contrast microscopy, recoiled into the uroid often leaving the original 'focal contact' behind on the glass (Fig. 1c).

Neither 'associated contacts' nor the formation of 'focal contacts' could be discerned by conventional light microscopy. The existence of 'focal contact' filopodia predicted by interferometry was however confirmed by scanning electron microscopy (Fig. 1d).

As $10 \mathrm{~mm}-\mathrm{KCl}$ was perfused into the chamber the interference colour of the 'associated contact' changed in a matter of seconds from whitish-grey to black (Fig. $1 b$ ). Normal movement of the amoebae was not obviously hindered by $\mathrm{KCl}$ at this concentration. Re-irrigation of the chamber with deionized water led to a reversal in the interference colour from black through grey and an eventual return to white (Fig. 1c). This reversible change in the interference colour of the 'associated contacts' could be followed for several cycles with individual cells. When the 'associated contacts' were black the focal contacts could no longer be distinguished but their presence could be inferred from the continuing production of trailing filopodia. On return to deionized water the focal contacts were clearly visible (Fig. 1c).

By using a series of $\mathrm{KCl}$ solutions $(50 \mu \mathrm{M}$ to $0 \cdot 1 \mathrm{M})$ in the perfusion chamber and monitoring the response of individual cells it was just possible to detect a departure from the 

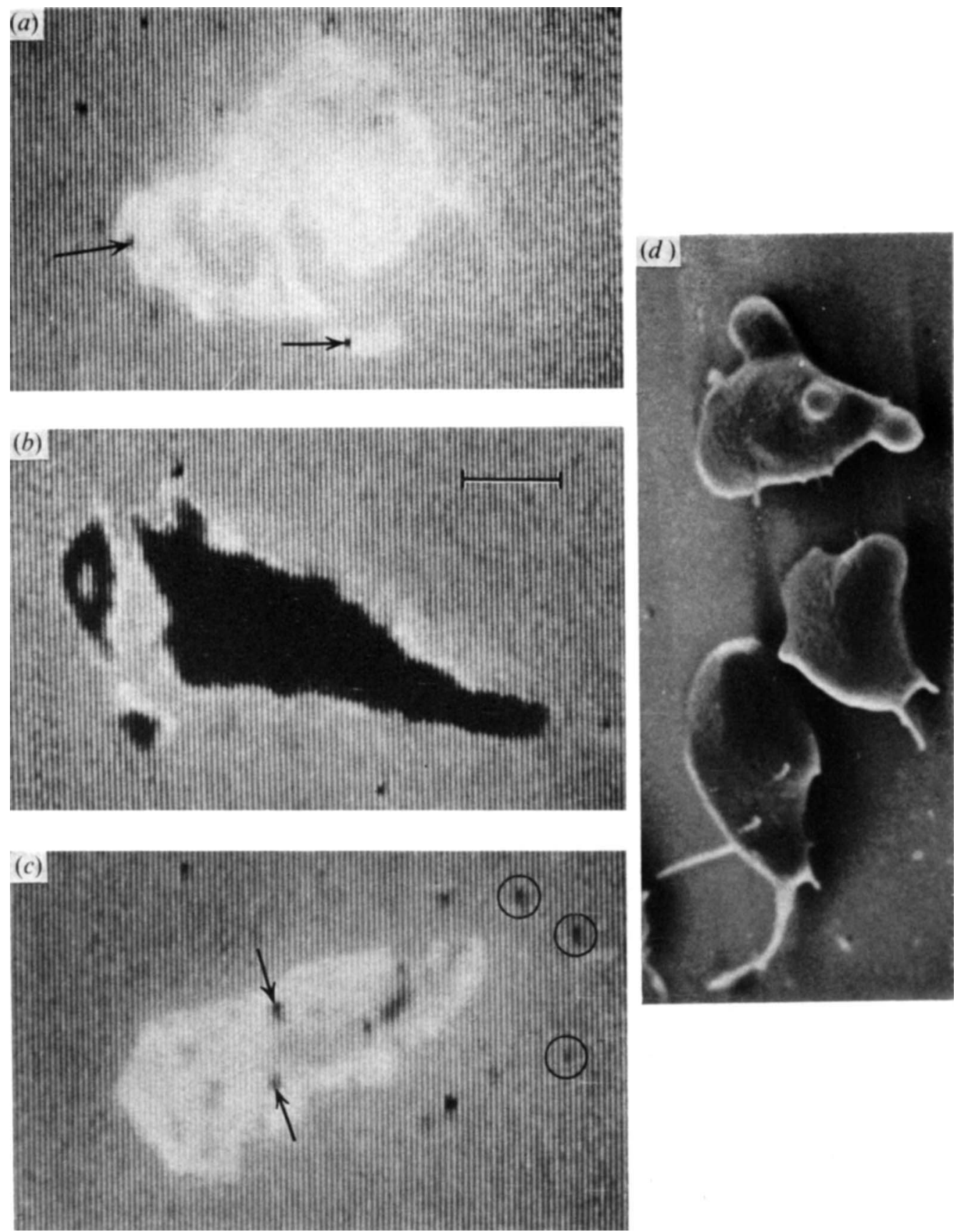

Fig. 1. $(a, b, c)$ Reflexion interference micrographs of Naegleria gruberi amoebae showing the contact behaviour of the undersurface on glass in response to changes in the ionic composition of the medium. Only those parts of the ventral surface close to the glass can be seen, i.e. regions of the ventral surface at distances greater than $150 \mathrm{~nm}$ from the glass are not seen. (Bar marker represents $5 \mu \mathrm{m}$.)

(a) Amoeba in deionized water; two black 'focal contacts' (arrowed) are visible within the white areas of 'associated contact' where the cell-substrate distance is about $110 \mathrm{~nm}$.

(b) The same cell in $10 \mathrm{mM}-\mathrm{KCl}$; the 'associated contacts' are now black (i.e. the cell-substrate distance has been reduced to not greater than $20 \mathrm{~nm}$ ).

(c) The same cell returned to deionized water; 'focal contacts' (arrowed) again become visible under the area of 'associated contact' and also remnants of these 'focal contact' points (circled) have been left behind on the glass beyond the rear end of the advancing cell.

(d) Scanning electron micrograph of $N$. gruberi amoebae. Note the relatively smooth outline of the cells, which are linked to the glass by slender filopodia. (Approximate magnification $\times 2500$.) 
'associated contact' interference colour that was generated in deionized water when the $\mathrm{KCl}$ concentration reached $400 \mu \mathrm{M}$. Since addition of sucrose up to $20 \mathrm{mM}$ final concentration failed to perturb the zero-order pattern, we are confident that the $\mathrm{KCl}$ effect does not result from changes in tonicity or refractive index. When experiments were carried out using $\mathrm{NaCl}, \mathrm{CaCl}_{2}$ and $\mathrm{La}\left(\mathrm{NO}_{3}\right)_{3}$, alteration in the interference pattern occurred in a similar way to that described for $\mathrm{KCl}$ (Preston \& $\mathrm{King}$, unpublished).

By reference to the interference colour series we could assign a value to the optical path difference $\Delta$ between the 'associated contact' regions of Naegleria amoebae and the glass surface of about $220 \mathrm{~nm}$ in deionized water. Perfect destructive interference was not obtained under our conditions, therefore it was impossible to assign an accurate value to the black image generated by the 'focal contacts' in deionized water and by the 'associated contacts' in $10 \mathrm{mM}-\mathrm{KCl}$; an estimate of $40 \mathrm{~nm}$ was considered to be an upper limit. The objective lens was set to an I.N.A. of 1.0, therefore the physical separation between the general undersurface of Naegleria amoebae ('associated contacts') and glass is roughly $110 \mathrm{~nm}$ in deionized water and not greater than $20 \mathrm{~nm}$ in $10 \mathrm{~mm}-\mathrm{KCl}$. These values were obtained using the formula (Izzard \& Lochner, 1976): gap width $=\Delta / 2 n \cos \theta$, where $\theta$ is the angle of refraction $\left(41^{\circ}\right)$ in the electrolyte medium and $n$ is the refractive index of that medium.

Electrophoretic measurements (Forrester, Gingell \& Korohoda, 1967) showed that Naegleria amoebae carry a net negative surface charge of the same order of magnitude as that borne by the more widely studied mammalian cells. Therefore amoebae moving on glass, a negative surface, could be susceptible to electrostatic repulsive forces which can act between bodies of like charge (i.e. as in the maintenance of lyophobic colloids). These long-range forces can be modulated by alteration of the charge density of the opposed surfaces and by changes in the ionic conditions of the intervening medium. If these forces were operating between a moving amoeba and the glass, the gap separating them should decrease with an increase in ionic strength of the medium; this change should be reversible on dilution of the electrolyte. Curtis (1964) found this to be the case with avian fibroblasts. The results of our perfusion experiments with Naegleria amoebae also concur with this prediction. Since the surface ${ }^{-}$charge density of these amoebae is not known we cannot put an accurate value on the thickness of the electrical double layer that would surround them under the ionic conditions of our experiments. Nevertheless, the dimensions of this layer can be expected to alter considerably because while the Debye length $(1 / K)$ for $200 \mu \mathrm{M}-\mathrm{KCl}$ is about $20 \mathrm{~nm}$ it reduces to about $3 \mathrm{~nm}$ in $10 \mathrm{mM}-\mathrm{KCl}$ (see Shaw, 1970).

The value of not greater than $20 \mathrm{~nm}$ for the 'focal contact' separation from glass in deionized water derived from interferometry is undoubtedly too large. Experiments employing surfaces coated with lectin films (T. M. Preston \& D. S. O'Dell, unpublished) demonstrate clearly that Naegleria amoebae can adhere to a substrate by means of antigenantibody type bonding. Therefore direct molecular attachment to a surface by means of 'focal contacts' borne on filopodia cannot be ruled out during amoeboid movement. However what we do question is the necessity for contacts with such an exact specificity for the normal progression of Naegleria. Indeed, major modifications of the surface properties of Naegleria achieved by capping off the peripherally located binding sites for specific antibodies and concanavalin A (Preston, O'Dell \& King, 1975) or cationized ferritin (King \& Preston, 1977) result neither in failure of 'focal contacts' to develop nor in disruption of amoeboid movement.

The relationship of a moving amoeba with its substrate can thus be seen as a two step process. Firstly, at a considerable distance from the substrate 'associated contact' is established. Secondly, from this broad platform, filopodia emerge ventrally to make the stable adhesions ('focal contacts') to the substrate that are essential for translational movement of the amoeba. The demonstration that in deionized water the gap of about $100 \mathrm{~nm}$ between the ventral surface of actively moving cells and glass can be dramatically and reversibly modulated by small changes in ionic strength is, we believe, biological 
evidence for the involvement of long-range electrostatic repulsive forces in maintaining at least the 'associated contact' during amoeboid locomotion of Naegleria.

We thank Drs D. Gingell and M. A. Rosemeyer for helpful discussions, and S. Pittman and A. Wiffen for technical assistance. This work was supported by a grant from the Science Research Council. The S.E.M. services used were provided by University of London, Zoology Board of Studies.

\section{REFERENCES}

BeLL, L. G. E. \& JEON, K. W. (1963). Locomotion of Amoeba proteus. Nature, London 198, 675-676.

Curtis, A. S. G. (1964). The adhesion of cells to glass: a study by interference reflection microscopy. Journal of Cell Biology 19, 199-215.

Curtis, A. S. G. (1973). Cell adhesion. Progress in Biophysics and Molecular Biology 27, 315-386.

Dellinger, O. P. (1906). Locomotion of amoebae and allied forms. Journal of Experimental Zoology 3, 337-358.

Forrester, J. A., Gingell, D. \& Korohoda, W. (1967). Electrophoretic polarity exhibited by amoeboid cells of Naegleria gruberi. Nature, London 215, 1409-1410.

HABEREY, M. (1971). Bewegungsverhalten und Untergrundkontakt von Amoeba proteus. Mikroskopie 27, 226-234.

IzZARD, C. S. \& LochneR, L. R. (1976). Cell-tosubstrate contacts in living fibroblasts: an interference reflection study with an evaluation of the technique. Journal of Cell Science 21, 129-159.

King, C. A. \& Preston, T. M. (1977). Fluoresceinated cationised ferritin as a membrane probe for anionic sites at the cell surface. FEBS Letters 73, 59-63.

Preston, T. M., O'Dell, D. S. \& King, C. A. (1975). Fluorescence microscope observations of some surface components of the amoeboflagellate Naegleria gruberi during amoeboid locomotion. Cytobios 13, 207-216.

SHAw, D. J. (1970). Introduction to Colloid and Surface Chemistry, 2nd edn, p. 139. London: Butterworths.

Wohlfarth-Botterman, K. (1973). Compte rendu de la table ronde numéro 10: mouvements améboides. IV Congrès International de Protozoologie, Clermont-Ferrand 1973. Cytobiologie 8, 194-202. 